\title{
Enunciación
}

\section{Pedagogía de la lectura en educación popular: el caso de los pre-ICFES populares en Bogotá}

\author{
Pedagogy of reading in popular education: \\ a case study of popular pre-ICFES in the city of Bogotá
}

Jazmín Amparo Márquez Ortiz*

\section{Resumen}

Este artículo se deriva de una investigación desarrollada en el marco de la Maestría en Pedagogía de la Lengua Materna, de la Universidad Distrital Francisco José de Caldas. En él se analizan las prácticas lectoras adelantadas por dos pre-ICFES de educación popular, cuyo trabajo se orienta a la democratización del acceso a la educación superior en Bogotá. El marco de estudio se ubica en el enfoque sociocrítico y discursivo de la lectura. Por medio del diseño de estudio de caso colectivo y el uso estrategias como el análisis de documentos, la entrevista a profundidad y la observación narrativa, se interpretan las prácticas lectoras en los dos casos y se identifican: a) fundamentos teóricos y pedagógicos de la lectura en la educación popular; b) la perspectiva didáctica de la lectura en las prácticas, y c) las relaciones existentes entre las prácticas lectoras y las necesidades de las comunidades. De esta manera, los resultados de la investigación evidenciaron que las prácticas lectoras inciden significativamente en la trayectoria pedagógica y social de los pre-ICFES de educación popular. Asimismo, se determinó que dicha incidencia se instala en dos planos -instrumental y esencial-, los cuales expresan la tensión entre lo institucional/hegemónico y lo popular/contrahegemónico.

Palabras clave: lectura, lector, texto, educador, educación popular, pre-ICFES populares.

\begin{abstract}
This document is the product of an investigation developed in the frame of the postgraduate program of Master in Mother Tongue Pedagogy at the Universidad Distrital Francisco Jose de Caldas. Here, reading practices developed in two "pre-ICFES" popular education initiatives, in which the work was orientated to the democratization of the access to advanced education in Bogota were analyzed. The study frame was located in the social, critics and discursive perspective of the reading. Using the case study design and strategies like the documents analysis, deep interviews and narrative observation, reading practices were interpreted in the two popular education initiatives with the aim of describe: i) theoretical basics and pedagogy in the reading in the popular education; ii) reading didactic perspective in the practice; and iii) possible relations between reading practices and communities requirements. In such a way, results evidenced that reading practices significantly affect pedagogy and social actions course in the "pre-ICFES" popular education. Moreover, it was determined that the incidence is settle in two planes -instrumental and essentialthat express the tension between institutional/hegemonic and popular/counter-hegemonic practices.
\end{abstract}

Keywords: reading, reader, text, educator, Popular Education, pre popular ICFES.

* Licenciada en Humanidades y Lengua Castellana de la Universidad Distrital Francisco José de Caldas; magíster en Pedagogía de la Lengua Materna de la misma universidad. Docente con experiencia en el campo de la educación popular y la educación formal. Miembro de la Fundación Sinéstesis. Actualmente, docente del proyecto Maestro Itinerante de la Universidad Nacional Abierta y a Distancia. Correo electrónico: jamarquezo@correo.udistrital.edu.co

Cómo citar: Márquez Ortiz, J.A.(2020). Pedagogía de la lectura en educación popular: el caso de los pre-ICFES populares en Bogotá. Enunciación, 25(1), 91-102. DOI: https://doi.org/10.14483/22486798.16014

Artículo postulado: 10 de marzo de 2020; aprobado: 26 de mayo de 2020 


\section{Introducción}

La educación popular ha generado significativos aportes al análisis y discusión de la lectura desde una perspectiva social y crítica. Así, la tradición pedagógica popular ponderó la lectura como potencial herramienta para desarrollar en los sujetos populares una conciencia crítica del orden social dominante y del papel que la educación bancaria (Freire, 1970) ejerce en favor de dicho orden.

La lucha por el acceso igualitario a la lectura -y a la escritura- como requisitos para la participación democrática, se erigió en el pilar político y pedagógico que ha orientado el trabajo de la educación popular por mucho tiempo. En el caso particular de Colombia, las diferentes organizaciones de educación popular han trabajado comprometidamente por la reducción del analfabetismo en niños, jóvenes y adultos no escolarizados. Sin embargo, la baja calidad educativa, así como los inferiores índices de profesionalización, y por tanto de empleabilidad, han demostrado que las necesidades educativas de la sociedad van mucho más allá de la simple alfabetización.

De la misma forma, en diferentes escenarios sociales y académicos se ha demostrado que la lectura no concluye en la decodificación de signos lingüísticos. Por el contrario, esta actividad del lenguaje supone el desarrollo de competencias necesarias para participar y gozar de los beneficios de un mundo cada vez más globalizado y, paradójicamente, con menos oportunidad para las personas del tercer mundo.

Desde esta perspectiva, y en una tensión constante con la escuela tradicional, han surgido en Colombia los pre-ICFES populares -en adelante PIP-, orientados a preparar a jóvenes y adultos para presentar las Pruebas de Estado Saber 11; un filtro importante que define el ingreso a las universidades públicas del país.

Los PIP surgen de diferentes movimientos y organizaciones sociales preocupados por "la exclusión que el sistema educativo superior ejerce sobre el grueso de la población perteneciente, principalmente, a sectores populares de la sociedad" (Picón y Mariño, 2016, p. 156). Una exclusión que se instala en los escenarios de mayor vulnerabilidad, donde los jóvenes que no obtienen los resultados necesarios para ingresar a universidades públicas deben adquirir deudas con altas tasas de interés para pagar sus estudios en instituciones de naturaleza privada. Otros, en muchos casos, simplemente no consiguen acceder a la educación superior, limitando sus posibilidades de ascenso social, cultural y económico.

Esta problemática ha visibilizado la dimensión de las desigualdades educativas en Colombia. De acuerdo con el Sistema Nacional de Información de la Educación Superior (SNIES), solo la mitad de los jóvenes colombianos entre los 17 y 21 años acceden a un establecimiento de educación superior. Cabe aclarar que en su mayoría ingresan a institutos universitarios o tecnológicos, y no a universidades propiamente. Además, tan solo 38,7 \% de estos jóvenes logra hacer un tránsito inmediato del grado $11^{\circ}$ a la educación superior; mientras que la taza de deserción es de 9 \% (SNIES, 2020).

En suma, la vasta brecha educativa del país que se hace perceptible, no solo en el acceso desigual a la educación superior, sino además en la baja calidad de la educación básica impartida, constituye uno de los principales desafíos de los PIP.

En este contexto tuvo lugar la presente investigación, cuyo objetivo fue determinar la incidencia de las prácticas lectoras en los PIP adelantados por dos colectivos de educación popular en la ciudad de Bogotá.

La lectura etnográfica con la que se dio inicio al estudio indicó que la necesidad de atender a los requisitos institucionales establecidos por el Instituto Colombiano para la Evaluación de la Educación (ICFES) a través las pruebas estandarizadas Saber 11, ubica a los PIP en una tensión constante entre lo hegemónico y lo contrahegemónico. Dicha tensión se expresa, por ejemplo, en el desequilibrio existente entre las percepciones de los educandos y las concepciones, apuestas teóricas y saberes pedagógicos de los educadores. 
Así, la concepción de los educandos con respecto a la lectura resalta su dimensión prescriptiva, entendida esta como un medio para lograr el dominio y el uso correcto de la lengua; los educadores, por su parte, declaran una postura ecléctica en donde conviven dos concepciones de la lectura. Por una parte, relacionan esta actividad del lenguaje con el desarrollo del pensamiento crítico y la capacidad de los sujetos para actuar e incidir en el contexto social y cultural; y por otra, anclan la definición de lectura al conjunto prestablecido de habilidades técnicas y gramaticales (Caraballo, 2014).

Para delimitar esta problemática, se rastreó un grupo de 26 antecedentes investigativos desarrollados entre los años 2012 y 2018, en países como Chile, Argentina, Colombia y España. Estos antecedentes ubicaron el estado de las investigaciones relacionadas con el presente estudio, al alrededor de tres temáticas: perspectivas o enfoques teóricos de la pedagogía de le lectura en educación popular; experiencias relacionadas con la enseñanza de la lectura en educación popular; y, tensiones político-pedagógicas presentes en contextos educativos populares.

Las investigaciones rastreadas dieron cuenta de la vigencia del debate en torno a lo tradicional vs. lo emergente. Así, reflexionan sobre el riesgo que atraviesan las iniciativas de educación popular de cara a las prácticas pedagógicas tradicionales y prescriptivas. Dicho de otro modo, estas investigaciones problematizan algunos factores que pueden inducir a contradicciones entre los principios e intencionalidades de la educación popular, y las prácticas concretas adelantas por las organizaciones en los distintos territorios.

A partir de lo expuesto, el presente documento examina los fundamentos teóricos y pedagógicos de la lectura en el marco sociocrítico de la educación popular. Asimismo, analiza las perspectivas didácticas presentes en las prácticas lectoras desde la caracterización del lector, el texto y el educador. Con ellos, se pretende dar luces sobre los sentidos y contradicciones que instituye la pedagogía de lectura en los PIP, de cara a las necesidades de los jóvenes y adultos.

\section{Marco conceptual}

Este análisis se enmarca en el paradigma crítico de la educación popular. Por su carácter dinámico, derivado de las múltiples configuraciones que adopta en los territorios en que se desarrolla, es difícil brindar una definición universal de la categoría educación popular. Sin embargo, Torres (2013) se refiere a ella como a "un movimiento pedagógico y una corriente sociocultural" cuyo significado se precisa a través de sus implicaciones políticas (p. 17).

La anterior noción se complementa con los núcleos comunes -o aspectos convergentes entre las diferentes experiencias de educación populardescritos por Torres (2013): a) la lectura crítica del orden social vigente y del papel que ha desempeñado allí la educación formal; b) el horizonte político emancipador en contra del orden social excluyente; c) la intención de contribuir al empoderamiento de los sectores históricamente dominados, y d) la búsqueda de metodologías coherentes con los valores transformadores de la educación popular desde el diálogo y la participación activa.

La educación popular, entonces, se orienta a la formación de sujetos capaces de leer críticamente su realidad social y educativa, empleando para ello "procedimientos específicos, propuestas metodológicas, y dispositivos de saber-poder-acción" (Mejía, 2014 , p. 9). Esto, con el fin de promover iniciativas colectivas que transformen las condiciones de opresión sufridas diferencialmente por los sectores menos favorecidos de la sociedad. No obstante, en este paradigma educativo crítico, lo popular hace referencia, más que a una clase social, a su contribución en la formación de sujetos históricos.

Ahora bien, desde sus inicios, con el desarrollo del modelo de alfabetización crítica, y a lo largo de su evolución teórico- práctica, la educación popular ha ponderado a la lectura como eje de 
reflexión e intervención desde una perspectiva histórica, sociocultural y crítica.

Desde esta mirada, la lectura es concebida como una práctica individual y colectiva encauzada por el conjunto de habitus o principios generadores que organizan las acciones y representaciones en torno a esta. Dichos habitus, que a veces pasan desapercibidos, varían según las condiciones de existencia particulares de cada sujeto o grupo social (Jasso et al., 2017). Así, la lectura como cualquier otra práctica humana se enraíza en un marco social específico, y su sentido responde a necesidades y propósitos relevantes dentro de este marco.

Al no encontrarse separada de otras prácticas sociales y culturales, se deduce que no existe una definición unívoca sobre la lectura. Por el contrario, geográfica, cultural e históricamente coexisten diversas lecturas que se alojan en una pluralidad de géneros discursivos; y que por lo demás, obedecen a los imaginarios, ideologías y creencias de determinado grupo social. Al respecto, Cassany (2015) señala que la lectura no es una actividad neutra, puesto que "leer es un verbo transitivo" que se expresa en las múltiples, dinámicas, y concretas prácticas que tienen lugar en cada comunidad humana.

Un aspecto de interés es el modo en que la educación popular ha cuestionado el dominio de la escuela tradicional sobre las prácticas lectoras. Así, se considera que la escuela impone un modelo hegemónico, universalista y excluyente sobre lo que significa leer. Esto ha permitido legitimar la noción de lectura como actividad de adiestramiento, individual, silenciosa y pasiva. Para Jurado (1995), desde esta perspectiva no existe un sujeto lector, sino "un sujeto retransmisor de un discurso que se le impone como verdad y que conduce a la abulia intelectual" (p. 70). En este mismo sentido, Sawaya y Cuesta (2016) analizan el uso escolar de la lectura como un medio de dominación y concluyen que "la escuela selecciona los textos, privilegia formas de lectura y de interpretación, sin cuestionar ni evidenciar los objetivos, los intereses de quién y al servicio de quién están" (p. 15).
Si bien, la lectura es una herramienta de dominación, puede ser a su vez un medio para la liberación de los sujetos. Desde esta lógica, Freire (1981) define la lectura como "un acto de conocimiento y un acto creador" (p. 6), por medio del cual los sujetos analizan el mundo y el lugar que ocupan en él. La finalidad del acto lector como proceso de liberación, plantea Freire, es llegar a "la razón de ser [...] un conocimiento cada vez más científico de su quehacer y de su realidad" (p. 64).

De acuerdo con lo expuesto, se definieron algunos elementos básicos para analizar las prácticas lectoras. Como se observa en la tabla 1, la categoría lectura es abordada desde tres subcategorías a las que, a su vez, les corresponden algunos descriptores específicos.

El lector, en primer lugar, es caracterizado como un sujeto cuyas capacidades le permiten no solo comprender literalmente un texto, sino además establecer relaciones, analizar el contexto, develar las representaciones implícitas y tomar postura (Cassany, 2015). Este lector crítico, además de enfrentar al texto con una actitud irreverente (Giroux, 2000, citado por González, 2006), actúa sobre sí y sobre su entorno generando transformaciones concretas. Para Freire (1991), el lector establece una relación dialéctica que compromete al discurso crítico con la acción transformadora sobre el mundo.

Dado que el acto lector no se reduce a la descodificación del lenguaje escrito, "sino que se anticipa y se prolonga en la inteligencia del mundo" (Freire, 1981, p. 1), la definición de la subcategoría texto tampoco puede acotarse al código lingüístico en su sentido tradicional. Más que eso, el texto es entendido como el conjunto de signos que se tejen para dar forma y sentido a la realidad, en lo que Freire denominó la palabra-mundo (1991).

Una característica inherente al texto como constructo social es la diversidad que se expresa en los múltiples formatos y géneros discursivos. De esta manera, los textos toman forma y contenido según las necesidades e intencionalidades de las personas en los diferentes contextos (Jurado, 2008). 
Tabla 1. Categorías de análisis

\begin{tabular}{|c|c|c|}
\hline \multirow{14}{*}{ Lectura } & S.C. & Descriptores \\
\hline & \multirow{7}{*}{ 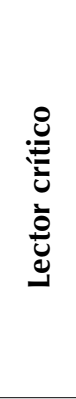 } & $\begin{array}{l}\text { 1. Identifica el género discursivo y/o el formato en que se presenta un texto para inferir su intencionalidad } \\
\text { comunicativa. }\end{array}$ \\
\hline & & $\begin{array}{l}\text { 2. Reconoce el valor de cada unidad y su relación con otros elementos para la construcción de un sentido } \\
\text { global del texto. }\end{array}$ \\
\hline & & 3. Analiza el texto de cara a su contexto histórico, social y cultural de producción. \\
\hline & & 4. Establece relaciones intertextuales de carácter lingüístico y semiótico. \\
\hline & & 5. Identifica las representaciones y puntos de vista implícitos en un texto. \\
\hline & & 6. Toma postura y expresa sus valoraciones, juicios e inquietudes sobre el texto. \\
\hline & & 7. Actúa en coherencia con la postura que asume desde las diferentes lecturas. \\
\hline & \multirow{3}{*}{ 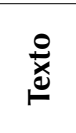 } & 8. Los contenidos expresados en los textos permiten problematizar la realidad de los lectores. \\
\hline & & 9. Se emplean variedad de textos que responden a condiciones hipermodales (imágenes, videos, pinturas). \\
\hline & & 10. Se emplean textos pertenecientes a diversos géneros discursivos. \\
\hline & \multirow{3}{*}{ 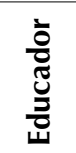 } & 11. Emplea estrategias didácticas para el desarrollo de la intertextualidad lingüística y semiótica. \\
\hline & & 12. Orienta al lector para desentrañar los contenidos implícitos del texto. \\
\hline & & 13. Guía discusiones grupales que ponen en diálogo la diversidad de puntos de vista sobre la lectura. \\
\hline
\end{tabular}

Fuente: Márquez (2019).

Finalmente, con respecto a la caracterización de la subcategoría educador, vale la pena citar la postura de Torres (2013), quien sintetiza muy bien el horizonte formativo de la educación popular. Educar, señala Torres, "es conocer críticamente la realidad, educar es formar sujetos para la transformación de la realidad, y educar es diálogo" (p. 31). En este marco, el educador implementa la lectura con el fin de desarrollar el pensamiento crítico de sus educandos mediante el diálogo y la intertextualidad como estrategias didácticas para la construcción y negociación de saberes contextualizados.

\section{Metodología}

El estudio se inscribe en el método de investigación cualitativo, entendido como "un conjunto de prácticas interpretativas que hacen al mundo visible, lo transforman y convierten en una serie de representaciones en forma de observaciones, anotaciones, grabaciones y documentos" (Neiman y Quaranta, 2006, p. 17). Asimismo, se instala en el paradigma interpretativo en tanto busca la comprensión del mundo de la vida desde la mirada de quienes lo experimentan (Vasilachis, 2006).

El diseño de investigación seleccionado fue el estudio de caso colectivo o de interpretación colectiva (Simons, 2011), el cual se centra en la definición de un problema y "la elección de varios casos para describirlo, analizarlo e ilustrarlo, con el objetivo de mostrar sus diferentes perspectivas desde la lógica de la réplica" (Hernández, 2014, p. 19).

Dentro del ámbito educativo, este diseño de investigación es valioso, por cuanto permite abordar "realidades educativas complejas e invisibilizadas por la cotidianeidad" (Álvarez y San Fabián, 2012, p. 4), lo que ayuda a entender sus dinámicas, contradicciones y dilemas. Por esta razón, se consideró que el estudio de caso colectivo era el camino adecuado para explorar un contexto educativo diverso, alternativo y territorial como es el de la educación popular.

La selección de los casos a analizar atendió a algunos criterios que aseguraban la sistematicidad 
de la investigación. De una parte, se buscaron iniciativas cuyas prácticas estuvieran vinculadas a la enseñanza y el aprendizaje de la lectura; se procuró que dichas iniciativas contaran con al menos cinco años de experiencia en educación popular; y se valoró la apropiación y arraigo a los diferentes territorios geográficos y culturales.

De acuerdo con estos criterios, fueron seleccionados para el estudio dos colectivos de educación popular: los pre-ICFES populares Los 12 juegos y También el viento. Estos colectivos se encuentran integrados por jóvenes universitarios y profesionales de Bogotá, quienes coinciden en la intencionalidad de democratizar el acceso a la educación superior.

El caso 1, pre-ICFES popular Los 12 juegos, es una iniciativa adelantada desde el año 2010 en el salón comunitario Chipacuy, ubicado en el barrio Compartir de la localidad de Suba. Está conformado por un grupo de treinta jóvenes; sin embargo, solo seis de ellos forman parte del equipo base, es decir del grupo que direcciona el proceso. Los restantes son educadores itinerantes que participan esporádicamente.

Al pre-ICFES asiste un significativo pero inconstante número de estudiantes pertenecientes a los estratos 1, 2 y 3, y provenientes, en su mayoría, de colegios públicos de Suba.

El nombre Los 12 juegos alude a un fragmento de la canción El baile de los que sobran, en donde se expresa: "El futuro no es ninguno / de los prometidos en los doce juegos. A otros les enseñaron / secretos que a ti no. A otros dieron de verdad esa cosa Ilamada educación". Esta canción es referente del pre-ICFES porque manifiesta el rechazo ante las desigualdades del sistema educativo hegemónico, y su impacto en el orden social excluyente.

El caso 2, pre-ICFES popular También el viento, debe su nombre al Festival del Viento y la Cometa que se lleva a cabo en la localidad de San Cristóbal, sector en donde se adelanta el pre-ICFES desde el año 2013.

Esta iniciativa ha abierto sus puertas a jóvenes de colegios públicos de estratos socioeconómicos
1 y 2, pertenecientes a la zona alta de localidad de San Cristóbal. Se encuentra constituido por un equipo base de cuatro educadores, profesionales y estudiantes de últimos semestres de distintas áreas del conocimiento, cuyo quehacer parte de una "lectura crítica de la sociedad, de la educación predominante y del sistema educativo colombiano, específicamente de la inequidad que lo caracteriza" (También el viento, 2019).

Cabe resaltar que la estructura curricular, para los dos casos, se organiza desde los componentes evaluados en las pruebas estandarizadas: lectura crítica, sociales, matemáticas y ciencias naturales. Asimismo, las clases se adelantan los sábados y domingo con el fin de no interferir en las actividades escolares de los participantes.

Ahora bien, para llevar a cabo el estudio de caso colectivo se emplearon tres instrumentos de recolección de información. De una parte, la entrevista a profundidad indagó sobre las concepciones y experiencias de los educadores en relación con la enseñanza y el aprendizaje de la lectura. De otra, a través de la observación narrativa descriptiva de las clases se profundizó en el quehacer, más precisamente en las acciones concretas que se desarrollan con respecto a la enseñanza de la lectura, y que expresan las apuestas didácticas de los educadores. Por último, el análisis de documentos brindó información sobre los planteamientos epistemológicos y lineamientos pedagógicos que orientan la pedagogía de la lectura.

Con relación al procedimiento para examinar los datos, se establecieron dos niveles de análisis. El primero, de codificación abierta, permitió "diseccionar, fragmentar, segmentar y desenmarañar los datos" (Hernández, 2014, p. 196), para caracterizar la presencia y recurrencia de la categoría lectura en los dos casos. Posteriormente, en el nivel axial se agruparon "las piezas para crear conexiones entre categorías y temas" (Hernández, 2014, p. 456). Es decir, se realizó la triangulación de la información obtenida en el nivel anterior determinando los factores compartidos y diferenciados entre los casos. 


\section{Análisis y discusión de resultados}

Siguiendo la metodología descrita, se determinaron los hallazgos que se discuten a continuación.

\section{El lector}

Se identificó que el enfoque teórico de las prácticas lectoras vehiculiza dos concepciones disímiles del lector.

De una parte, los documentos y las observaciones, principalmente, dieron cuenta de una perspectiva cognitiva y netamente lingüística desde la cual se reduce el rol del lector al desarrollo de las habilidades y competencias evaluadas en la Prueba Saber 11. De esta manera, se enfatiza en el fortalecimiento de los niveles de lectura literal e inferencial, considerando que la mayoría de los educandos que Ilegan a los pre-ICFES presentan dificultades para interpretar los elementos literales de un texto.

Así, se identificó en los pre-ICFES la intención de fortalecer "las habilidades de lectura que en el colegio no se han desarrollado a cabalidad" (PCLC, 2018). Los PIP asumen como su misión el reto de responder a la baja calidad educativa que los estudiantes han recibido a lo largo de su vida, y cuyos síntomas se expresan en numerosas dificultades lectoras.

La concepción del lector descrita anteriormente se adhiere a la finalidad urgente de los PIP, es decir, la necesidad inmediata de incidir en los resultados de las Pruebas Saber 11, para que los jóvenes logren acceder a la educación superior (Martínez, 2017). Esto les implica a los pre-ICFES el reto de reconocer y negociar con contenidos y habilidades que, con respecto a la lectura, son legítimos en el marco institucional del ICFES y de la educación formal.

Por otra parte, se identificó una concepción sociocrítica del lector desde la cual es concebido como un sujeto reflexivo y transformador, capaz de analizar e incidir en su realidad educativa y social. Al respecto, los educadores exponen que:
[...] aprender a leer desde la educación popular tiene una propuesta política que tiene que ver con cómo leemos lo que nos pasa, cómo leemos nuestra vida, cómo leemos el mundo [...] lo crítico está en eso, cómo esa lectura ayuda a develar el orden oculto, las cosas que hay que cambiar. (Entrevista 1, C1 También el viento, 2018)

El anterior enunciado resalta el carácter diverso, político y contrahegemónico que esta actividad del lenguaje adquiere en el marco de la educación popular. Desde la mirada de los educadores, la lectura se considera una actividad transversal a la vida de los sujetos que no solo se instala en los escenarios tradicionales de enseñanza/aprendizaje como la escuela. Por el contrario, los fenómenos más cotidianos son susceptibles de ser leídos; razón por la cual el lector se torna un intérprete del mundo.

Esta perspectiva, se asocia a lo que Martínez (2017) define como la dimensión fundamental de los PIP, haciendo referencia a aquellos elementos que se enmarcan en la apuesta ética y política de la educación popular, y que son imprescindibles para lograr acción transformadora (p. 215).

\section{El educador}

Al indagar sobre la perspectiva didáctica en la enseñanza de lectura pudo determinarse que las acciones de los educadores son eclécticas y transitan constantemente entre lo tradicional y lo crítico.

Por una parte, en el desarrollo de las clases se privilegió el uso didáctico del diálogo a fin de fortalecer en los educandos la intertextualidad y la capacidad de leer tras las líneas del texto/realidad. De esta manera, se identificó que en los pre-ICFES es fundamental la búsqueda de metodologías coherentes con el principio de construcción horizontal y colectiva del conocimiento, como la lectura colectiva, mesas redondas, discusiones, debates, socializaciones, entre otras.

En esta misma dirección, se resalta la intención de los educadores de desarrollar el pensamiento crítico en sus educandos para que estos puedan 
"develar, problematizar y darle vuelta a lo establecido, a lo que se presenta como 'conocimiento universal' aportar en la recuperación de los saberes ausentes que no aparecen en los libros de texto ni en las pruebas estandarizadas" (EMAR, 2018). Esto permite entender que, para los educadores, la labor no se agota en facilitar el ingreso de los jóvenes a la universidad; por el contrario, guiados por un espíritu irreverente, esperan que la lectura crítica sea una posibilidad de escapar a la imposición de verdades absolutas

De otro lado, se develaron algunos aspectos que refuerzan la didáctica tradicional de la lectura en los pre-ICFES. Por ejemplo, los educandos que asisten a los PIP son estudiantes de instituciones educativas distritales que pasan la mayoría de su tiempo allí, por tanto, es difícil transformar los habitus escolares que regulan las concepciones y prácticas de estos sujetos con respecto a la lectura.

Adicionalmente, no siempre se cuenta con un número suficiente de educadores que pueda responder a la cantidad de estudiantes. Por la misma razón, en muchas ocasiones no hay docentes especializados en el área del lenguaje, así que deben encargarse de las clases educadores formados en otras áreas del conocimiento o profesionales sin experiencia pedagógica previa. Esto genera la presencia de tendencias diversas con respecto a la enseñanza de la lectura e incluso contradictorias con los principios pedagógicos de la educación popular.

Por último, son reducidos los espacios colectivos de planeación y evaluación curricular, por cuanto apremian aspectos de orden logístico que demandan bastante tiempo. De esta manera se limitan las posibilidades del quehacer, pues no se reflexiona conjuntamente sobre los aciertos o desaciertos del desarrollo pedagógico. Asimismo, la inconstancia en la asistencia de los estudiantes genera obstáculos para realizar una evaluación integral del proceso.

\section{El texto}

El uso didáctico de los textos, para los dos casos guardó estrecha relación con aspectos evaluados en las Pruebas Saber 11. Así, las tipologías textuales abordadas se relacionan con la clasificación de textos continuos y discontinuos que se expresa en la tabla 2, tomada de uno de los programas curricular del componente de lectura crítica.

Con respecto al texto, los educadores insisten en la importancia de que el lector pueda diferenciar y comprender la diversidad de géneros discursivos, a partir del análisis de su forma y de su finalidad comunicativa.

Asimismo, en el desarrollo de las clases y en las entrevistas destacó la intención de que los contenidos de los textos problematicen la realidad de los educandos. En este sentido, los educadores sugieren "hacer una buena selección cultural de textos para que sean atractivos, pertinentes a la realidad social de los chicos" (E2C1, 2018). Asimismo, exponen que "al ser la lectura la decodificación de cualquier tipo de signos [...] puedan leer no sólo textos, sino todo tipo de fenómenos sociales" (E3C1). Así, se abordaron temáticas tales como el miedo, el barrio, proyectos de vida, la espiritualidad, la sexualidad, entre otros que forman parte del texto-realidad: el tejido de las experiencias vitales y sociales de los lectores.

Tabla 2. Tabla de tipologías textuales

\begin{tabular}{lll}
\hline & \multicolumn{1}{c}{ Literarios } & Informativos (descriptivos, expositivos, argumentativos). \\
\hline Continuos & $\begin{array}{l}\text { Novela, cuento, poesía, } \\
\text { canción, dramaturgia. }\end{array}$ & Ensayo, columna de opinión, crónica. \\
\hline Discontinuos & Caricatura, comic. & Etiqueta, infografía, tabla, diagrama, aviso publicitario, manual, reglamento. \\
\hline
\end{tabular}

Fuente: Documento 1, C1 pre-ICFES popular de Suba (2018) 


\section{Los pre-ICFES populares: entre lo esencial y lo instrumental}

Con todo lo dicho, se advierte que la incidencia de la lectura en los PIP se expresa en dos tendencias importantes. De una parte, incide en un plano instrumental caracterizado por la intención de responder a las necesidades concretas e inmediatas de la comunidad. Para los pre-ICFES, la intención instrumental es minimizar las desigualdades en el acceso a la educación superior que afectan diferencialmente a los jóvenes habitantes de las localidades de Suba y San Cristóbal.

En esta dirección, los PIP tienen como uno de sus objetivos instrumentales, fortalecer las habilidades lectoras que la escuela formal no ha logrado desarrollar en los educandos. Esto, con el fin de que los jóvenes alcancen al menos un nivel básico de lectura que les permita responder adecuadamente a las Pruebas Saber.

Por otra parte, en su plano esencial, la lectura se encuentra en función de propiciar el pensamiento crítico y el empoderamiento de los educandos respecto a sus propios proyectos de vida, y a los de su comunidad. Esto es, lograr que cada sujeto se reconozca y actúe como parte de un colectivo que sueña, lucha y se transforma en beneficio de todos.

Desde el plano esencial de los pre-ICFES, no se lee solo para obtener buenos resultados en las pruebas estandarizadas. De fondo, el sentido de la lectura como acto creador está en la posibilidad de cuestionar y transformar las condiciones de injusticia por las cuales se ha marginado a los sujetos populares.

Lo anterior representa una tensión constante por el riesgo de que las acciones, concepciones y saberes de los educadores en torno a pedagogía de lectura se concentren en responder a las demandas institucionales del ICFES, entrando en contradicción con la perspectiva sociocrítica que adquiere la lectura en la educación popular. Del mismo modo, la intención de formar lectores críticos y transformadores no puede avasallar la necesidad inmediata que tienen los educandos de prepararse para las
Pruebas Saber 11, siendo esta la razón por la cual acuden a dichos espacios de formación.

En tal sentido, el reto de los PIP es lograr la convergencia entre los planos instrumental y esencial. Por lo cual la pedagogía de la lectura deberá estar en función de facilitar el ingreso a la universidad, y al mismo tiempo, permitir la formación de profesionales con alto sentido de pertenencia y capacidad crítica para comprometerse ética y políticamente con el desarrollo social, cultural, económico, y político de sus territorios, desde cualquier área del conocimiento.

En suma, los planos instrumental y esencial consiguen conectarse en tanto el acceso a la educación superior aporte al mejoramiento de las condiciones de vida materiales y culturales, no solo de los individuos, sino además de la comunidad a la cual pertenecen.

\section{Conclusiones}

Las prácticas lectoras inciden significativamente en el desarrollo de los PIP; su influencia es determinante para el logro de las acciones pedagógicas y sociales de estas dos experiencias de educación popular, que logran un impacto importante en el mejoramiento de las condiciones de vida individuales y colectivas en los territorios, a partir del empoderamiento de los sujetos populares.

En los casos estudiados coexisten dos finalidades de la lectura ubicadas en planos distintos, y determinadas a partir de la caracterización del lector, el texto y el educador. Por un lado, la finalidad instrumental centrada en el fortalecimiento de las competencias y habilidades lectoras evaluadas en las Pruebas Saber 11. Por otra parte, la finalidad esencial de formar lectores críticos y conscientes de la realidad social y de su papel como sujeto histórico. Estas dos tendencias se traducen en una tensión constante entro lo institucional/hegemónico y lo popular/contrahegemónico.

Didácticamente, los pre-ICFES privilegiaron estrategias lectoras basadas en el diálogo, el pensamiento crítico, y la intertextualidad, respondiendo 
al principio popular de la construcción colectiva y significativa del conocimiento. Sin embargo, se identificaron algunos aspectos que afectan negativamente el quehacer de los educadores, como: la necesidad de responder a los requisitos de las Pruebas Saber 11; el reducido número de educadores; la predominancia de habitus lectores prescriptivos en los educandos; la falta de formación de los educadores en el campo de la pedagogía crítica y del lenguaje; la inconstancia en la asistencia de los educandos; las limitaciones de tiempo y de espacios destinados a la reflexión pedagógica; entre otros. Es así como la pedagogía de la lectura en los pre-ICFES populares adopta tendencias disimiles y contradictorias, al tiempo que induce a la predominancia del plano instrumental sobre el plano esencial.

Finalmente, se destaca la importancia de continuar explorando las prácticas y sentidos pedagógicos de las experiencias de educación popular, puesto que, siendo escenarios complejos que se instalan al margen de la institucionalidad, aportan nuevas perspectivas y dilemas al estudio de la pedagogía de la lectura.

\section{Reconocimientos}

Artículo derivado de la investigación realizada para optar por el título de magíster en Pedagogía de la Lengua Materna, Universidad Distrital Francisco José de Caldas.

\section{Referencias bibliográficas}

Álvarez, C. y San Fabián, J. (2012). La elección del estudio de caso en investigación educativa. Gazeta de Antropología, 28(1). Recuperado de http://www. gazeta-antropologia.es/?p=101

Caraballo, R. (2014) Formación para el empleo y educación popular. [Tesis de doctorado]. Universidad de Sevilla. Sevilla. Recuperado de https://dialnet. unirioja.es/servlet/tesis?codigo $=44924$
Cassany, D. (2015). Literacidad crítica: leer y escribir la ideología. Recuperado de https://docplayer.es/10121443-Literacidad-critica-leer-y-escribir-la-ideologia.html

Freire, P. (1970). Pedagogía del oprimido. Montevideo: Tierra Nueva.

Freire, P. (1981). La importancia del acto de leer. Trabajo presentado en la apertura del Congreso Brasileño de Lectura. Campinas, Sao Paulo.

Freire, P. (1991). La importancia de leer y el proceso de liberación. México: Siglo XXI Editores.

González Martínez, L. (2006). La pedagogía crítica de Henry A. Giroux. Revista Electrónica Sinéctica, 29, 83-87. Recuperado de https://www.redalyc.org/ pdf/998/99815739014

Hernández, R. (2014). Metodología de la investigación. México: McGraw-Hill.

Jasso Velázquez, D., Villagrán Rueda, S., Rodríguez Ortiz, M., Aldaba Andrade, M., Calvillo Ríos, C. y Acosta de Lira, J. (2017). Habitus, práctica y lectura escolar. Abordaje cualitativo. Revista de Educación Superior, 1(1) 12-28. Recuperado de https://www. ecorfan.org/republicofperu/research_journals/Revista_de_Educacion_Superior/vol1 num1/Revista_ de_Educaci\%C3\%B3n_Superior_V1_N1_2.pdf

Jurado, F. (1995) Lectura, incertidumbre, escritura. Forma y Función, 8, 67-74. Recuperado de https:// revistas.unal.edu.co/index.php/formayfuncion/ article/view/16967/17835

Jurado, F. (2008) La formación de lectores críticos desde el aula. Revista Iberoamericana de Educación, 46. Recuperado de https://rieoei.org/historico/documentos/rie46a05.htm

Márquez, J. (2019). La lectura y la escritura en tres colectivos de Educación Popular en la ciudad de Bogotá. Un estudio de caso. [Tesis de maestría]. Universidad Distrital Francisco José de Caldas. Bogotá.

Martínez, N. (2017) Del río al caracol: transformando la cotidianidad. Sistematización de la experiencia de la Pre-Universidad Tunjuelo Popular (2012-2016). [Tesis de maestría]. Universidad Pedagógica Nacional. Bogotá. 
Mejía Jiménez, M. (2014). La educación popular: una construcción colectiva desde el Sur y desde abajo. Education Policy Analysis Archives, 22, 1-31. Recuperado de https://www.redalyc.org/ pdf/2750/275031898079.pdf

Neiman, G. y Quaranta, G. (2006). Los estudios de caso en la investigación sociológica. En I. Vasilachis de Gialdino (comp.), Estrategias de investigación cualitativa (pp. 213-237). Barcelona: Gedisa.

Picón, P. y Mariño, J. (2016). Pre-ICFES y preuniversitarios populares en Colombia Educación popular. Aportes, 60, 153-175.

Pre-ICFES popular de Suba Los 12 juegos (2016). Declaración Constitutiva II. Recuperado de https://preicfespopulardesuba.wordpress.com/
Simons, H. (2011). El estudio de caso: teoría y práctica. Madrid: Morata.

Sistema Nacional de Información de la Educación Superior (SNIES) (2020). Indicadores de educación superior. Bogotá. Recuperado de https://www.mineducacion.gov.co/sistemasdeinformacion/1735/ w3-article-212350.html?_noredirect=1

Torres, A. (2013). La educación popular latinoamericana: contextos y desafíos actuales. Revista Pueblos, 56, 28-30. Recuperado de http://www.revistapueblos.org/blog/2013/05/15/la-educacion-popular-latinoamericana-contextos-y-desafios-actuales/

Vasilachis, I. (2006). Estrategias de investigación cualitativa. Barcelona: Gedisa. 
Anexo 1. Tablas de codificación de fuentes

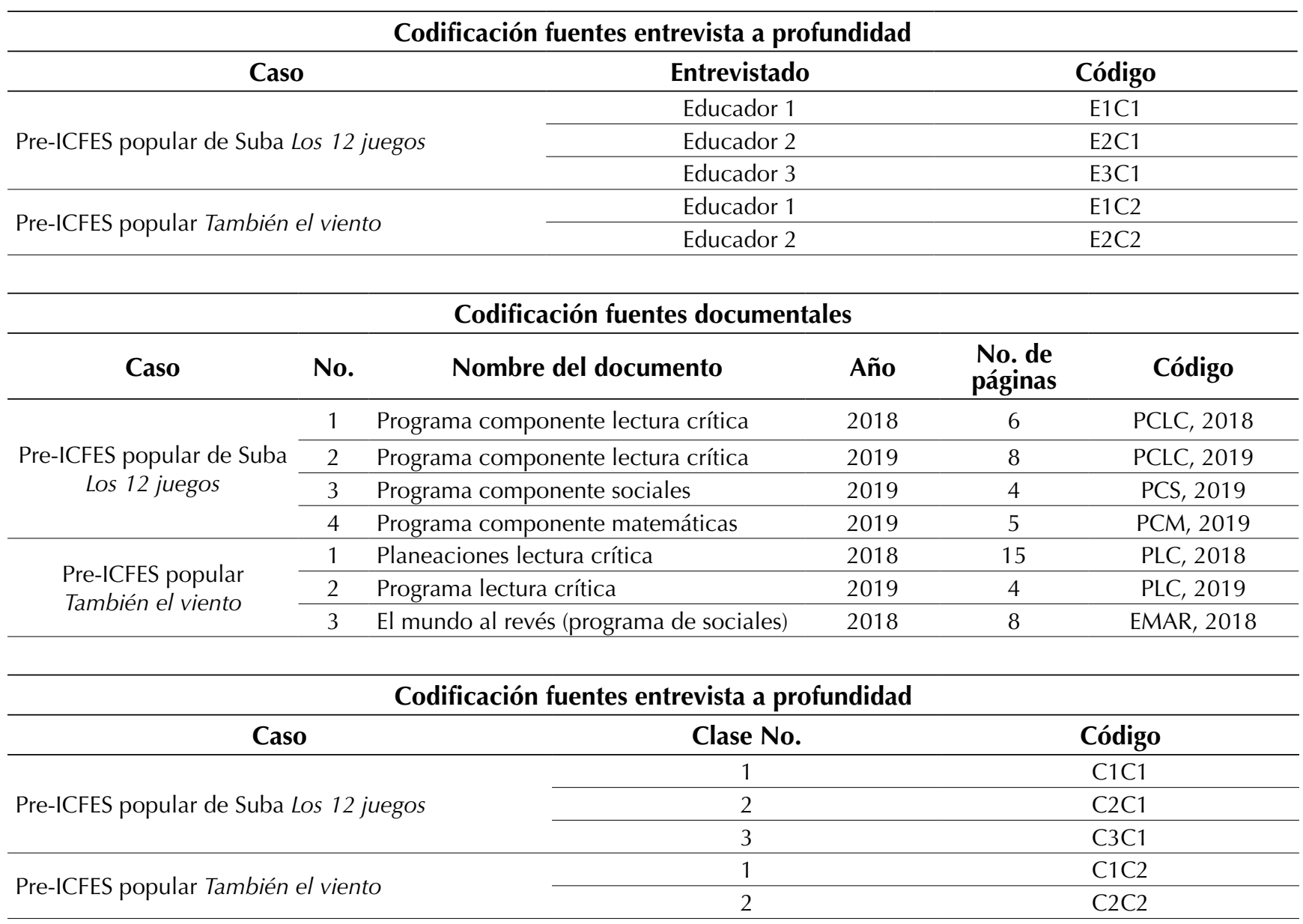

